Meta

Journal des traducteurs

Translators' Journal

\title{
De la traduction comme acte créateur : raisons et déraisons d'un déni
}

\section{Jean-Yves Masson}

Volume 62, numéro 3, décembre 2017

La traduction littéraire comme création

URI : https://id.erudit.org/iderudit/1043954ar

DOI : https://doi.org/10.7202/1043954ar

Aller au sommaire du numéro

Éditeur(s)

Les Presses de l’Université de Montréal

ISSN

0026-0452 (imprimé)

1492-1421 (numérique)

Découvrir la revue

Citer cet article

Masson, J.-Y. (2017). De la traduction comme acte créateur : raisons et déraisons d'un déni. Meta, 62(3), 635-646. https://doi.org/10.7202/1043954ar d'utilisation que vous pouvez consulter en ligne.

https://apropos.erudit.org/fr/usagers/politique-dutilisation/ 


\title{
CODA
}

\section{De la traduction comme acte créateur: raisons et déraisons d'un déni}

\author{
JEAN-Y VES MASSON \\ Université Paris-Sorbonne* \\ jymasson.htlf@gmail.com
}

\section{Introduction}

Que la traduction ne soit pas «créatrice», c'est le lieu commun le plus répandu à son sujet, justifiant le mépris millénaire dans lequel elle a été tenue en Occident. On se souvient du dialogue entre un géomètre et un traducteur dans les Lettres persanes de Montesquieu:

«Il y a vingt ans que je m'occupe à faire des traductions. - Quoi! Monsieur, dit le géomètre, il y a vingt ans que vous ne pensez pas? Vous parlez pour les autres, et ils pensent pour vous?» (Lettre $\left.\mathrm{n}^{\circ} 129\right)$.

On aurait tort de croire que cet échange, qui revient apparemment à une condamnation de la traduction, reflète au premier degré la pensée de Montesquieu lui-même: il s'agit plutôt d'une sorte de mise en abîme discrète et ironique de la nature même des Lettres persanes, dont l'auteur (tout en gardant l'anonymat) se présente dans son introduction, ne l'oublions pas, comme le simple traducteur. L'original des Lettres est bien entendu censé être en persan, et lui-même, pour les traduire, prétend les avoir dérobées à l'attention de leurs véritables auteurs. Comme il s'agit là d'une fiction que Montesquieu ne cherche même pas à rendre crédible, aucun lecteur ne peut se tromper sur le fait que les Lettres persanes sont une pseudo-traduction qui masque en réalité un original.

Ce qu'a voulu l'auteur des Lettres persanes avec ce préambule, c'est bien sûr souligner l'artifice qui fait le génie de son livre: donner à voir son propre pays par les yeux d'étrangers qui s'étonnent de ce qui paraît tout naturel aux autochtones. Mais c'est donc qu'il s'agit aussi pour lui d'utiliser le français tout en y faisant résonner une pensée venue d'ailleurs, obéissant à des modes de pensée qui ne sont pas français. Il s'agit d'introduire de l'étranger dans la langue, d'écrire comme s'il traduisait une langue étrangère. Bien sûr, le faux traducteur ne manque pas, pour rassurer son lecteur, de dire qu'il a coupé certaines tournures par trop orientales; les Lettres pourront donc se lire «comme un livre français»... Montesquieu, sous son masque, feint 
ainsi de remplir le "pacte de lecture» que tout traducteur, à l'époque classique, est tenu de conclure avec son public: on attend de lui qu'il adapte (bien que le mot n'existe pas encore dans ce sens!) en ôtant tout ce qui peut choquer ou ennuyer le bon goût français. Ainsi s'explique le fait qu'Uzbek et ses compatriotes, bien qu'étant persans, s'expriment comme de parfaits gentilshommes parisiens.

Mais n'est-il pas évident que Montesquieu entend ainsi souligner que s'il était vraiment traducteur, justement, il ne resterait pas passif devant le texte à traduire? Loin de s'abstenir de penser, comme le croit le géomètre de la lettre 129, il interviendrait même massivement, comme c'était si souvent l'usage à son époque, en n'hésitant pas à retrancher des pans entiers du texte. Cervantès, dans Don Quichotte, autre traduction fictive (l'original du livre, rappelons-le, est censé être un manuscrit rédigé en arabe), a mis en œuvre le même genre de rappels périodiques, indiquant dans certains chapitres qu'il retranche les longueurs de la narration initiale.

Mais le plus important n'est pas là: la fiction qu'emploie Montesquieu sert à introduire dans l'esprit du lecteur une distance relativement au texte qui permettra d'excuser les façons de penser «barbares» d'un étranger amené à émettre sur la France des affirmations qu'on ne tolérerait peut-être pas de la part d'un Français. L'œuvre étrangère est ainsi plus «naturellement» scandaleuse pour le lecteur qui ne saurait complètement s'offusquer que des Persans n'aient pas les façons de penser de son propre pays. Montesquieu, bien sûr, n'y est pour rien : il n'est, dit-il dans sa tentative particulière de captatio benevolentioe, «que» le traducteur.

C'est qu'il est bien rassurant, mais de toute évidence faux, de séparer traduction et création comme deux sphères qui ne communiqueraient pas. Un livre, affirme la doxa, est écrit par son auteur, puis traduit par des traducteurs, qui donc ne sauraient faire œuvre «originale» car ils n'en ont pas le droit. L'évidence première qu'il existe un texte premier et un texte second - c'est-à-dire, comme dirait Monsieur de La Palisse, qu'il faut, pour traduire un livre, que celui-ci ait d'abord été écrit - est ainsi subrepticement transposée, transcrite en une distinction entre "original» et «copie», le traducteur devant s'abstenir, prétend-on, de toute forme de «création» s'il veut rendre justice à l'œuvre qu'il traduit. Le traducteur doit «s'effacer» jusqu'à devenir invisible: cet impératif rejoint l'idéal bien connu de la traduction «transparente» qui se fait oublier comme telle. Or, nous le savons bien, cet effacement n'est jamais absolu. N'est même jamais complètement possible: faute de pouvoir s'effacer (car les mots qu'il choisit pour traduire sont bien les siens et non ceux d'un autre traducteur, qui livrerait un résultat différent), le traducteur cherche à se faire oublier, ce qui est loin d'être la même chose. Et qui n'est peut-être jamais tout à fait possible non plus: tel est l'objet de la présente réflexion.

\section{Le régime fiduciaire de la traduction}

On ne saurait trop rappeler que la naissance d'une véritable pensée de la traduction, dont les prémisses se trouvent dans le romantisme allemand, et qui allait devenir la «traductologie» au $\mathrm{xx}^{\mathrm{e}}$ siècle, a coïncidé avec la prise de conscience de l'insuffisance des stéréotypes traditionnels que l'on vient de rappeler, et avec le désir de réhabiliter le traducteur en montrant que son rôle relativement à un texte ne saurait être passif. Mais jusqu'où vont les droits du traducteur? Est-il un esclave au service de l'œuvre qu'il traduit? Les mêmes qui lui dénient tout droit à se prétendre créateur seront 
souvent les premiers à trouver mauvaise une traduction «servile», à savoir le «mot à mot» que la tradition occidentale presque unanime refuse depuis Cicéron. C'est pour éviter les apories auxquelles on est rapidement conduit par une pensée de ce genre qu'ont été choisis les termes de «source» et de "cible» qui évitent le registre imprécis et suspect de l'«originalité». La question de la création dans la traduction apparaît alors sous un angle nouveau et peut être formulée ainsi: quelle part d'auctorialité le traducteur possède-t-il dans l'objet «traduction»?

Cette question n'est pas simple à résoudre et elle touche même à ce qui dérange les historiens de la littérature et les critiques dans une traduction. Monsieur de La Palisse, là aussi, a son mot à dire, et ce mot est loin d'être sans intérêt: si on lui demande de quoi le traducteur est au juste l'auteur, il répondra qu'il est l'auteur... de sa traduction. En tant qu'objet littéraire, un livre traduit a en réalité deux auteurs: l'auteur du texte source et le traducteur en tant qu'il est «l'auteur» du texte cible. Dont pas un mot, certes, n'aurait pu être écrit sans le texte premier, dont pas une phrase n'existe par elle-même mais seulement en référence à une ou plusieurs... phrases du texte source, mais sans que je ne puisse jamais cerner avec certitude la part subjective qui revient au traducteur. Pour commencer à apprécier cette part, il faut comparer (au moins) deux traductions du même texte par deux traducteurs différents: les différences qui apparaissent alors, et qui ne peuvent manquer d'apparaître, manifestent cette part de subjectivité du traducteur qui entre dans la composition du texte que nous lisons, sans qu'il s'agisse forcément d'erreurs commises par l'un des deux. Subjectivité ne veut pas dire non plus arbitraire: si chaque traducteur a sa propre manière de comprendre le texte, c'est qu'il est d'abord un lecteur. Et nous savons depuis Proust qu'un livre contient autant de livres différents que ce livre aura de lecteurs: personne n'y voit tout à fait la même chose, bien que tout le monde lise le même livre.

Pourtant, le statut normal, le régime ordinaire de la traduction n'est pas de s'offrir à une telle lecture comparée. Quand je lis une œuvre traduite, la totalité des mots que je lis ont été écrits par le traducteur; plus un seul mot, par définition, n'est «de» l'auteur. On n'en finit pas de se pencher sur l'abîme qu'ouvre ce constat tout simple, tellement simple et tellement vertigineux que, les trois quarts du temps, il fait l'objet d'une complète dénégation (et j'emploie ce terme au sens que lui donne la psychanalyse, tout en remettant à plus tard le soin d'approfondir cette question). Car en dépit des enseignements qu'on peut tirer de la comparaison entre plusieurs traductions d'un même texte, il n'existe pas d'électrolyse permettant de séparer l'oxygène apporté par l'auteur de l'hydrogène que le traducteur a greffé sur lui. Le texte traduit est une eau que je bois sans pouvoir dire de qui elle me vient.

Manifestement, ce n'est pas là chose agréable à penser: tout le monde aspire à puiser à la source. J'ai donc intérêt, puisque je ne peux faire autrement (car la fonction première de la traduction est de s'adresser aux lecteurs qui n'ont pas la maîtrise de la langue source et doivent donc en passer par une traduction pour pouvoir accéder à l'œuvre), j'ai intérêt pour ma tranquillité d'esprit à oublier autant que faire se peut que ce que je lis n'est pas l'œuvre originale. Ce que je souhaite, c'est pouvoir penser (et dire) que j'ai lu Les Frères Karamazov. Seul un examen approfondi me révélera l'abîme qui sépare, pour n'en citer que trois, la première traduction d'HalpérineKaminski et Charles Morice (1888, d'ailleurs qualifiée d'adaptation par les traducteurs eux-mêmes) de celle de Boris de Schloezer (1929), puis celle-ci à son tour de la 
traduction d'André Markowicz (2002). Selon la traduction que j'ai choisie, je n'ai tout simplement pas lu la même œuvre: certes, l'histoire et les personnages sont les mêmes, mais ils ne parlent pas du tout le même langage et ne sont donc... pas les mêmes. Je choisis pourtant là un cas où le dernier traducteur, Markowicz, a exposé ses principes de traduction, et dénoncé la langue académique dans laquelle Dostoïevski avait été traduit avant lui comme ne correspondant pas à ce qu'était vraiment Dostoïevski en russe. Le lecteur qui ne peut vérifier par lui-même entend ces arguments, peut s'informer de ce débat, mais ne peut ni donner raison à Markowicz ni réhabiliter Boris de Schloezer en connaissance de cause. Il prend donc ce qu'on lui donne et doit accepter de se laisser guider par le traducteur : il doit lui faire confiance. Le régime de la traduction est un régime fiduciaire. Je ne peux faire autrement que de prendre les mots du traducteur pour argent comptant. M’en méfier ne m’amènera à rien car je ne puis m'en méfier en connaissance de cause - sauf si je suis en mesure de contrôler par moi-même la traduction, et alors je ne suis pas le lecteur «normal» de la traduction, je suis un pédagogue qui cherche à vérifier s'il peut recommander cette traduction à ses élèves, ou un confrère du traducteur qui cherche à évaluer son travail, ou un curieux pervers qui lit une traduction plutôt que de lire l'œuvre originale (et cette perversion, certes, est légitime). Et c'est pourquoi toutes les préfaces de traducteurs depuis qu'elles existent (la première qui ait été conservée étant le $D e$ optimo genere oratorum de Cicéron) sont aussi des efforts pour se concilier la bienveillance du lecteur: il faut que celui-ci adhère au texte qu'on lui soumet et accorde au traducteur sa confiance.

Mais cette confiance n'est sans doute jamais pleine et entière, et l'est sans doute d'autant moins que la conscience littéraire du lecteur est plus développée. Que le traducteur revendique sa part créatrice (il est intervenu sur le texte pour en faciliter la lecture) ou qu'il la nie (il a été rigoureusement fidèle à l'original), il ne peut entièrement lever le soupçon qui pèse sur lui. Le lecteur bute-t-il sur une aspérité, il accusera d'abord le traducteur de maladresse, alors qu'il n'en est peut-être rien et que le texte source, à cet endroit, présente une aspérité comparable à laquelle le traducteur s'est échiné à trouver un équivalent. Le traducteur sait qu'il sera le premier accusé si le lecteur a le sentiment que «quelque chose ne passe pas» dans la langue cible: et c'est pourquoi la tentation est grande de gommer ces aspérités, de «lisser» le texte pour ne pas inquiéter le lecteur. Ce qu'Antoine Berman (1985/1991 ; Berman, Granelle, Jaulin et al. 1985) interprétait exclusivement, à tort selon moi, en termes d'ethnocentrisme ${ }^{1}$, je le comprends d'abord par ce souci de se mettre à l'abri du soupçon toujours renaissant du lecteur à l'égard du traducteur. Les réflexes ethnocentriques ne viennent qu'en second lieu et ne sont qu'une conséquence de cette soumission du traducteur aux critères qui lui assurent, croit-il, une communication plus fluide avec le lecteur. Le soupçon n'est peut-être totalement levé que quand le traducteur se trouve, exceptionnellement, reconnu pour sa qualité littéraire: Amyot et Baudelaire sont en français les archétypes de ce genre de consécration. Je lis les Vies de Plutarque traduites par Amyot comme une œuvre française, car il est admis qu'elle fait partie de notre littérature. De même l'Homère de Pope appartient-il pleinement à la littérature anglaise, au point que l'avoir lu est une obligation pour tout lecteur anglais cultivé, sans qu'il ait à se demander dans quelle mesure Homère y trouve son compte. Ces traductions consacrées sont délivrées de tout soupçon parce qu'elles ont quasiment perdu le statut de traductions pour conquérir celui d'œuvres à part entière, et pleinement reconnues comme telles. 


\section{La créativité du traducteur : une réalité refoulée par l'histoire littéraire et la lexicographie}

Mais ce sont là des exceptions. Nuançons encore notre propos. En fait, plus l'œuvre à traduire est littéraire, plus le public auquel s'adresse la traduction est cultivé, et plus il est possible au traducteur de demander à son lecteur de faire preuve de patience: bien des «notes du traducteur» ont cette fonction de faire entrer le lecteur dans «l'atelier» du traducteur, lequel éclaire la difficulté pour son lecteur. Le point extrême de cette pratique est atteint dans les fascinantes traductions de la Bible par Henri Meschonnic ${ }^{2}$ : chaque volume est accompagné d'une masse foisonnante de notes où le traducteur fait partager au lecteur la réflexion qui l'a amené à adopter la traduction proposée. On entre dans le laboratoire du poète-traducteur, consultant les dictionnaires, réfléchissant sur les racines sémitiques, tâtonnant jusqu'à trouver $s a$ solution. Le texte est imprimé sans appel de notes, parce qu'il y en aurait partout: au lecteur de lire en faisant l'aller-retour entre le texte et les notes, s'il le souhaite, après avoir lu une première fois le texte pour lui-même. C'est ce dispositif singulier qui permet au traducteur de libérer sa créativité poétique: dans la traduction du passage de Genèse, 11 sur la tour de Babel, quand Meschonnic substitue au «confondons leurs langues» sa propre solution: «embabelons leurs langues» (Meschonnic 2002: 63), il invente un mot et joue, en poète qu'il est, de tous les possibles de la langue française. Processus critique exposé en note et processus créateur à l'œuvre dans la suite de décisions innovantes aboutissent ainsi à une recomposition complète du texte.

Dans les textes de plus faible valeur littéraire au contraire, jusqu'aux textes de très grande consommation, la demande du public est première: le «client»-lecteur est roi et l'impératif imposé par l'éditeur au traducteur est de fournir le texte le plus limpide, le plus «consommable» qui soit. C'est donc bien souvent dans des textes appartenant à la paralittérature que la créativité du traducteur sera sollicitée de la façon la plus évidente! Il n'est que de regarder par exemple l'inventivité dont ont fait preuve les traducteurs de Harry Potter dans toutes les langues pour le comprendre, et particulièrement en français, notamment dans la recréation des noms propres. La note de bas de page étant interdite (car le jeune public ne l'admettrait guère), tout doit être compris tout de suite.

Certains traducteurs pensent que c'est également la règle pour la traduction littéraire «de haut vol» et qu'il faut toujours s'interdire les notes de bas de page: je ne suis pas de cet avis, car il me semble qu'il est essentiel de s'adapter à la nature des œuvres, à leur degré d'éloignement culturel par rapport au lecteur de la langue cible, etc. Mais on vient de voir par l'exemple de Meschonnic qu'il ne faudrait pas penser que la créativité du traducteur diminuerait au fur et à mesure que la qualité littéraire du texte augmente! La réalité est bien plus complexe: la créativité du traducteur est sans doute moins admise comme telle quand l'œuvre à traduire bénéficie du statut de chef-d'œuvre, puisque le lecteur n'aime pas l'idée que l'on s'interpose entre lui et l'auteur qu'il souhaite comprendre par lui-même. La part créatrice du traducteur sera donc souvent minimisée volontairement, voire dissimulée par ce dernier. Mais il y a mille exceptions à cette affirmation: on attend d'un traducteur qui donne une nouvelle version d'Alice au pays des merveilles qu'il propose des solutions neuves, la traduction d'un tel livre (et de tous les livres et poèmes de Lewis Carroll en général) obligeant le traducteur à prendre des risques s'il veut que son travail ait de l'intérêt. 
Il nous faut donc admettre l'inquiétude fondamentale qui entoure la traduction et nous habituer à son statut d'objet littéraire incertain, mais essentiel. Cela conduira à en finir avec le refoulement dont elle est l'objet dans l'histoire littéraire, surtout en France, où aucune histoire de la littérature française n'a jusqu'ici jamais envisagé que les traducteurs puissent eux aussi avoir le statut d'auteurs. On écrit toujours seulement l'histoire des œuvres «originales». On peut évoquer parfois des imitations, mais presque jamais les traductions comme telles: tout se passe comme si elles n'avaient jamais joué le moindre rôle dans la vie littéraire des siècles passés. Quand on mentionne que tel auteur français a été «influencé» (ce mot magique, hélas, n’a toujours pas disparu) par des œuvres étrangères, on ne se demande jamais à quelles traductions il a eu recours et on fait comme s'il les avait toutes lues en langue originale: ce qui est parfois vrai, bien sûr, mais pas très souvent.

Cet «oubli» de la traduction résulte directement du déni de créativité qu’on lui oppose: le traducteur doit rester dans l'ombre, seuls les auteurs ont droit de paraître au-devant de la scène. Ce préjugé, qui n’a toujours pas été remis en cause en France par les historiens de la littérature (mais il en va déjà tout autrement dans plusieurs pays de la francophonie), ne règne pas au même degré dans les autres aires linguistiques et culturelles: les Allemands, par exemple, savent depuis longtemps qu'il leur est impossible d'écrire l'histoire de leur littérature sans tenir compte des traductions. Mais il est vrai que c'est parce que la langue allemande moderne a été forgée par une traduction, celle de la Bible par Luther, et que cette inscription de la traduction à la naissance même de la langue interdit tout refoulement, tout oubli. Il n'empêche que les autres littératures ne devraient pas non plus pouvoir nier le rôle que jouent les traductions dans la maturation et l'évolution de toute langue. Ce rôle, nous pouvons le constater de façon plus spectaculaire pour l'allemand: et il est bien possible qu'il varie selon les langues (une vaste enquête reste à mener). Mais seule une histoire générale des traductions permettra de le vérifier. Disons donc pour l'instant les choses sous la forme d'une double négation: il n'est probablement aucune langue de culture dans la maturation de laquelle les traductions n'ont joué aucun rôle.

La question de la langue est en effet essentielle quand on réfléchit au rôle créateur joué par les traducteurs. Car la vision erronée de l'histoire littéraire que j'évoquais trouve son prolongement direct dans les dictionnaires: et là encore, la situation diffère selon les pays. Les dictionnaires de la langue française qui recourent à des citations de grands auteurs pour illustrer les usages d'un mot ne puisent, depuis Littré, que dans le corpus de nos grands auteurs. On ne cite pratiquement jamais les emplois qui ont pu en être faits par des traducteurs. Si je regarde en tête du Grand Robert la «Liste des auteurs et des ouvrages cités ( je dispose de l'édition de 1980), je n'y trouverai qu'une seule traduction: comme il faut bien mentionner la Bible, on me précise que celle-ci est citée «d'après Lemaistre de Sacy, Crampon ou Segond». D'autres auteurs sont venus s'ajouter, au fil de l'élaboration du dictionnaire, au corpus initial; la liste en est donnée à la fin du dernier tome. Dans cette liste apparaît enfin le Plutarque d'Amyot, ainsi que l'ensemble des traductions de Perrot d'Ablancourt, après le nom duquel figure entre parenthèses une liste très sommaire des auteurs qu'il a traduits: «Cicéron, Tacite, Arrien, Xénophon, César, Lucien, Thucydide, etc.». Et... sauf erreur de ma part, c'est tout! Mieux que rien, donc: mais cela en dit long sur les traductions considérées comme recevables par la lexicographie au milieu du $\mathrm{xx}^{\mathrm{e}}$ siècle. La situation a très peu changé depuis. Et pour mesurer le contraste, il n'est 
que de consulter le Dictionnaire allemand des frères Grimm, monumentale description de la langue allemande dont le premier volume a paru en 1854 et qui n'a été achevée que dans la deuxième moitié du Xx ${ }^{\mathrm{e}}$ siècle (en 1961, avec un supplément en 1971): on constate qu'au contraire les plus éminentes traductions sont fréquemment citées au fil des articles, non seulement celles qui sont signées des plus grands auteurs (comme Schiller, Goethe, Wieland ou Schlegel), mais toutes celles qui ont joué un rôle éminent dans la constitution du patrimoine intellectuel de la langue allemande, même si leur "auteur» n'a pas acquis une importance comparable par ses œuvres personnelles: Johann Heinrich Voss, traducteur de Virgile et d'Homère, par exemple, ne peut être écarté d'aucune histoire littéraire et il est considéré comme un "auteur» par les lexicographes qui lui empruntent des citations. Plusieurs générations, en effet, ont lu Homère dans la traduction de Voss, ou ont appris à lire le latin de Virgile en s'aidant de sa traduction: c'est-à-dire qu'on a lu l'Homère de Voss, Homère comme une création de Voss. Création ou recréation, qu'importe! Selon la traduction qu'on lit, ce n'est pas au même Homère, au même Virgile que l'on a affaire. Il n'en va différemment dans aucune langue. Et il n'en va pas différemment pour les auteurs modernes et pour les classiques.

Sans doute est-ce pour cela que la littérature allemande offre plusieurs exemples d'auteurs pour lesquels il est impossible de séparer «œuvre personnelle» et «œuvre de traducteur»: le cas le plus éminent est sans doute celui de Friedrich Rückert, l'un des fondateurs de l'orientalisme allemand, qui étudia pas moins de 44 langues, en enseigna plusieurs à l'université, et dont les trois quarts de l'œuvre poétique sont composés de traductions de poèmes qui sont de véritables recréations, abondamment exploitées par les compositeurs allemands et autrichiens. L'idée de classer Rückert parmi les «simples» traducteurs ferait sourire avec condescendance n'importe quel Allemand cultivé; l'idée de nier que ses traductions, malgré leur qualité poétique, soient des traductions et de ne les considérer «que» comme des productions personnelles ne viendrait à personne; pas plus que celle de publier séparément ses poèmes «personnels» (dont certains, comme les Chants des enfants morts [Kindertotenlieder] mis en musique par Mahler, sont pourtant fort célèbres). Pourtant, en France, en 1989 encore, les éditeurs du premier tome des Euvres complètes de Gérard de Nerval dans la Bibliothèque de la Pléiade (Claude Pichois et Jean Guillaume) refusaient de publier les traductions de Nerval sous prétexte qu'il ne s'agissait pas de textes «de» lui: poussant le paradoxe, frustrant pour le lecteur, jusqu'à reproduire les articles de Nerval sur Heine en coupant les traductions des poèmes (et ne parlons pas des Poésies allemandes de 1829 dont n'est reproduite que la préface, excellemment annotée par Lieven D'hulst qui a dû souffrir de ne pouvoir éditer aussi les traductions ellesmêmes). Il est vrai que cette édition (par ailleurs remarquable) avait à faire le tri difficile entre les textes authentiques, ceux vraiment de la plume de Nerval, et ceux qui longtemps lui avaient été attribués par erreur, en raison des multiples pseudonymes auxquels il eut recours pour écrire dans la presse de son temps. Il n'empêche: jamais des traductions dues à un grand auteur ne devraient être traitées comme des apocryphes! Ce fut pourtant ce qui se produisit, dans cette édition qui, pour longtemps encore, fait autorité . $^{3}$ 


\section{Le traducteur, auteur second mais premier lecteur}

Il est donc temps (et je sais que je prêche ici des convertis, mais nous ne formons pas encore une bien grande église) de reconnaître que la traduction est une forme de création; une création seconde, certes, mais bien une forme de création, peu différente en cela de l'imitation et des multiples autres formes de réécriture qui ne se confondent pourtant pas, bien sûr, avec elle. Le statut créateur des autres formes de réécritures est plus facile à reconnaître, parce que la traduction est une réécriture sous contrainte constante, une re-création sur laquelle s'exerce à tout moment la présence contraignante d'un texte source qu'il faut en principe suivre pas à pas. En principe... car c'est loin d'être toujours le cas, l'adaptation (qui implique une prise de liberté) n'ayant pas perdu ses droits aujourd'hui encore, non seulement au théâtre, mais dans de nombreux autres domaines. Tout se passe donc comme si l'on identifiait création et liberté: d'où, je le crois profondément, la fascination pour l'autotraduction, à laquelle sont consacrées un nombre d'études savantes sans rapport avec son importance réelle, au moins du point de vue statistique ${ }^{4}$. Lautotraduction reste une exception, et si elle concerne des auteurs extrêmement importants comme Beckett ou Nabokov, ce n'est pas la seule raison pour laquelle la traductologie s'y intéresse tant. Ce qui fascine dans l'autotraduction, c'est que s'y trouve précisément éliminé ou plutôt courtcircuité ce que le régime «normal» (ordinaire) de la traduction a de plus troublant et de plus gênant: la présence du traducteur comme tiers qui s'interpose entre l'auteur et le lecteur, donc la source de cette méfiance consubstantielle à la traduction dont j'ai parlé plus haut. Or ce qui manque dans l'autotraduction, dans ce cas tout à fait exceptionnel de l'auteur devenu son propre traducteur, c'est justement cette contrainte que subit par définition le traducteur: l'auteur qui s'autotraduit ne se traduit pas, il se réécrit entièrement, libre de tout changer s'il le veut puisqu'il n'a de comptes à rendre qu'à lui-même. Nous voilà assurés d'avoir affaire, avec le même livre dans deux langues, à deux ouvrages de création "pure», que bien sûr nous pouvons comparer, mais sans avoir à redouter le péché d'infidélité, autrement dit, de liberté.

Le régime propre à la traduction est d'être une réécriture sous contrainte: et c'est, redisons-le, ce qui fait qu'on lui dénie sa nature créatrice. Mais depuis quand le fait qu'une écriture soit sous contrainte oblige-t-il à lui dénier toute valeur de création? Les contraintes sont depuis toujours une nécessité de la création littéraire, qu’elles soient fortement codifiées comme celles auxquelles se soumirent les troubadours provençaux, ou encore les Grands Rhétoriqueurs du $\mathrm{Xv}^{\mathrm{e}}$ siècle, ou de nos jours les écrivains de l'Oulipo - ou qu'elles soient plus subtiles, liées par exemple aux genres littéraires, aux règles de la prosodie, etc. et bien sûr aux règles que l'écrivain se fixe à lui-même.

Il y a en effet très peu de formes de création ex nihilo, c'est le sens des célèbres phrases de Proust (1927/1989) sur l'écriture comme traduction, dans Le Temps retrouvé; l'écrivain lui-même ne crée pas à partir de rien:

... je m’apercevais que, pour exprimer ces impressions, pour écrire ce livre essentiel, le seul livre vrai, un grand écrivain n'a pas, dans le sens courant, à l'inventer puisqu'il existe déjà en chacun de nous, mais à le traduire. Le devoir et la tâche d'un écrivain sont ceux d'un traducteur 5 .

Proust a créé son œuvre à partir de Mme de Sévigné, à partir de Saint-Simon, à partir de toutes les lectures qu'il a faites, à partir de Ruskin dont il a été le traducteur, 
à partir d'Anatole France (figure tutélaire de sa jeunesse), et de tous les autres écrivains qu'il a pu admirer, et bien sûr à partir de ce qu'il était lui-même, mais qu'il n'a pu découvrir que par la lecture puis par ces différentes formes de réécriture que sont la traduction (même si ce fut avec l'aide de sa mère), le pastiche, sans oublier la critique littéraire.

Le sujet que nous traitons, celui de la traduction littéraire, dans son rapport à la création, révèle donc ici des enjeux qui dépassent les questions relevant de l'histoire des idées, de la lexicographie, de l'histoire de la littérature ou de la langue. Ce sont des questions proprement philosophiques relevant de cette "traductosophie» que Jean-René Ladmiral, créateur de ce beau néologisme, appelle de ses vœux. Ce sont toutes les questions relatives à la tradition, et la parenté avec la traduction est évidente par la seule ressemblance entre les deux mots. En effet, nous ne nous créons pas nous-mêmes. Nous sommes créés par toute l'histoire qui nous fait, par les gens qui nous ont éduqués, par le pays auquel nous appartenons, les voyages que nous avons faits, les tableaux que nous avons vus, les films que nous avons aimés... et toutes les rencontres que nous avons faites, la rencontre d'un auteur depuis longtemps disparu pouvant être aussi importante dans une vie que celle d'un éducateur ou d'un ami. Aucun écrivain (et plus généralement aucun artiste) digne de ce nom ne devrait pouvoir nourrir le fantasme de la création ex nihilo, même si cela a existé dans l'histoire de la littérature: la table rase est toujours une illusion en matière de création artistique. Elle ne peut exister qu'en philosophie, mais le geste de Descartes ne vise que la vérité, non l'émotion, non tout ce qui relève de la sensibilité et dont il connaissait mieux que personne le prix. Et pour que Descartes rejetât tous les savoirs acquis depuis l'enfance, encore fallait-il qu'il les eût reçus: l'idée même du doute ne serait jamais venue à un homme inculte.

Symétriquement, elle ne serait jamais venue non plus à un penseur pétrifié de respect pour la tradition, pour la foi et les croyances héritées de ses ancêtres. Cela, encore, peut nous inciter à quelques réflexions sur la traduction dans ses affinités avec la tradition. Si la première traduction d'un texte est un acte courageux et risqué parce qu'il est inaugural, il est bien clair que, dès lors que ce texte est reconnu comme possédant une valeur primordiale, cette seule et première traduction ne saurait suffire; la retraduction est un des indices les plus certains de la canonisation d'un texte, et la multiplication des traductions contribue, dans toute culture, à la constitution du canon littéraire. Car ce canon ne saurait comporter uniquement des œuvres «nationales» et il n'est pas d'exemple de culture digne de ce nom qui ait été fermée à toutes les autres.

La traduction est donc dans une certaine mesure «sacralisante». Car l'une de ses premières fonctions est précisément de créer un canon: la traduction est créatrice de valeur, car on peut affirmer sans trop de risque que le fait de traduire un texte signifie qu'on lui attribue, qu'on lui reconnaît, une certaine valeur (pas forcément majeure, mais un certain intérêt, sans quoi on ne le traduirait pas). Et quand il existe d'un texte une multitude de traductions, quand un même texte a été retraduit cinq, dix, quinze, quarante fois, cent fois au fil des siècles, personne ne peut plus nier que ce texte fasse partie du canon: le recueil des cent traductions françaises de l'unique poème complet de Sappho réunies par Philippe Brunet ${ }^{6}$ en offre un exemple saisissant.

Mais la traduction est aussi, d'une autre manière, désacralisante: le traducteur qui retraduit un texte définit nécessairement son travail contre celui des traducteurs 
qui l'ont précédé. Si le premier traducteur occupe une place unique dans l'histoire de la réception d'un texte, c'est d'abord parce qu'il n'a personne contre qui prendre position, alors que tous ses successeurs se définiront contre lui. Ne serait-ce que dans sa volonté de tout reprendre à zéro, donc de «faire table rase» des traductions précédentes (ce qui n'est jamais complètement possible, mais le prouver nous entraînerait trop loin), dans l'insatisfaction qu'il éprouve à l'égard des versions antérieures, le «retraducteur» opère nécessairement un rajeunissement du texte. Fût-il vieux de trois ou dix ou trente siècles, le texte qu'on vient de traduire est tout neuf: il vient de (re)naître.

C'est ainsi que la vie d'un texte se poursuit dans les différentes langues à la manière d'une «création continuée» - poursuivie notamment de traduction en traduction, au fur et à mesure que les langues cibles changent, évoluent, et avec elles la sensibilité des lecteurs (le traducteur est l'un d'eux) qui de siècle en siècle trouvent à une même œuvre d'autres significations. Si la lecture est créatrice de sens, comme on l'a reconnu depuis longtemps, avant tout grâce à Proust, la traduction l'est au suprême degré dans la mesure où elle peut se définir comme une "écriture-lecture», et où toute traduction est la trace d'une lecture. Roland Barthes a présenté ce phénomène crucial pour la littérature dans un paragraphe de Critique et vérité qui passe malheureusement à côté du problème de la traduction:

Une œuvre est «éternelle», non parce qu'elle impose un sens unique à des hommes différents, mais parce qu'elle suggère des sens différents à un homme unique, qui parle toujours la même langue symbolique à travers des temps multiples: l'œuvre propose, l'homme dispose. (Barthes 1966: 52)

Cet «homme unique» dont parle Barthes, dont la théorie de la lecture à cette époque-là (1966) s'efforce encore de réduire le lecteur à une fonction sans subjectivité, n'existe assurément pas autrement que comme figure de pensée: la supériorité de Proust est de placer la subjectivité au cour de sa pensée. C'est pourquoi Proust se préoccupe de traduction, contrairement à Barthes, qui ne songe même pas ici que, pour parler «la même langue symbolique» d'âge en âge, l'homme parle avant tout et toujours une langue particulière qui n'est jamais «la» langue ni le langage. Non seulement le sens d'un texte ne cesse d'advenir et de se créer de lecteur en lecteur au fil du temps à l'intérieur d'une même langue, mais dans chacune des langues où le texte a été, est et sera traduit, ce sens s'enrichit, se développe, à travers la double subjectivité du traducteur et de ses lecteurs. Le traducteur est certes un «auteur second» mais il est aussi, d'un autre côté, un "premier lecteur» dont dépendront tous les autres lecteurs de sa traduction, laquelle entre en concurrence avec toutes celles qui l'ont précédée ou qui la suivront. La suggestion de «sens multiples » qu'opère tout texte au fil de l'histoire est l'œuvre des lecteurs, car la lecture elle aussi fait œuvre: la lecture est créatrice de sens. Mais il nous est permis de dire que la traduction, en tant que lecture, l'est au suprême degré. Le traducteur comme "premier lecteur» selon l'ordre chronologique, est aussi un «lecteur premier», au sens qualitatif du terme: il est probablement, avec le copiste (cette figure disparue du champ littéraire, mais qui y occupa si longtemps la première place), le seul lecteur qui aura prêté pleine et égale attention à chaque mot du texte et suivi l'auteur pas à pas comme son ombre, ou son double. Celui à qui, idéalement, la distraction n'est pas permise (même si, comme le copiste, il lui arrive de somnoler...), celui qui n'a pas le droit de sauter une ligne. 
Affirmer que la traduction n'est pas créatrice est donc une opinion à courte vue, une évidence que la réflexion invite à dépasser, comme l'ont fait les contributeurs de ces journées qui nous ont réunis à Avignon. Si nous parvenons, par nos travaux, à provoquer une prise de conscience de la nécessité de tenir compte de l'apport créateur des traductions, les conséquences en seront immenses et d'énormes chantiers vont s'ouvrir. Nous avons vu que les dictionnaires de la langue française étaient presque tous à refaire si l'on voulait tenir compte de ce qui est dû aux traducteurs dans le mouvement historique de la langue. Une autre conséquence sera probablement d'étudier la part des traductions dans la constitution du patrimoine intellectuel des différentes langues. C'est à ce projet que répondent, pour la langue française (bien au-delà des limites territoriales de la France, par conséquent), le projet de l'Histoire des traductions en langue française que j'ai l'honneur de codiriger avec Yves Chevrel, ainsi que, pour la langue anglaise, The Oxford History of Literary Translation in English dirigée par Peter France et Stuart Gillespie, qui obéit à des modalités légèrement différentes (notre Histoire ne se limite pas aux seules traductions «littéraires », même si literary translation inclut pour les auteurs de ce travail monumental davantage que la simple «littérature», notamment l'histoire et la philosophie). La notion de «patrimoine intellectuel» est celle qui nous a paru la plus appropriée pour désigner l'ensemble des traductions qui, dans tous les domaines du savoir, des connaissances techniques, mais aussi du divertissement, ont enrichi la langue en y important des ouvrages de toutes sortes, venus de l'étranger. Ce faisant, nous avons refusé la distinction opérée par Antoine Berman entre la «traduction des œuvres» (c'est-à-dire des textes littéraires où s'opère un véritable travail du style) et celle de ce qu'il avait coutume d'appeler la «parole creuse» : à leur façon, livres de piété ou manuels techniques font aussi partie du patrimoine d'une langue, et l'on en a beaucoup traduit au fil du temps!

Oui, il importe de réviser l'histoire littéraire des différents pays du monde, et de se souvenir que l'actualité littéraire d'une époque donnée a toujours été composée de traductions qui ont pu elles aussi «faire événement». Que les lecteurs ont toujours lu à la fois des livres «originaux» et des traductions. Que les écrivains sont aussi (peutêtre même d'abord) des lecteurs et que, grâce aux traductions, ils ont eu connaissance de littératures étrangères. Évidences? Sans doute, mais nous avons vu combien elles étaient refoulées. Une fois éclaircies, comme nous avons tenté de commencer à le faire ici, les raisons de ce refoulement, il devient urgent de le dépasser.

\section{REMERCIEMENTS}

Merci à Marine Charmasson et à Louisa Derdar qui ont transcrit la conférence dont le présent article est la refonte complète et, je l'espère, améliorée.

\section{NOTES}

* Centre de recherche en littérature comparée.

1. Berman, on le sait, oppose la traduction «ethnocentrique» occidentale à la traduction «éthique» qu'il appelle de ses vœux, où l'altérité de l'étranger est reconnue comme telle et non pas gommée.

2. Meschonnic, Henri (2002): Au commencement. Traduction de la Genèse. Paris: Desclée de Brouwer.

3. J’ai développé la critique que je résume ici dans la préface de mon édition des traductions poétiques complètes de Gérard de Nerval (De Nerval 1840/1996). 
4. Établissant en 2007 un «Bilan des recherches sur la traduction en littérature comparée» en France (Masson 2007: 67-79), j'avais été frappé par le nombre de thèses et d'essais sur l'autotraduction: une dizaine entre 1995 et 2005. S’y sont ajoutés, depuis, d'autres thèses et de nombreuses rencontres et colloques.

5. Proust, Marcel (1927/1989) : À la recherche du temps perdu. (Dirigé par Jean-Yves Tadié) Tome IV. Paris: Gallimard, 469.

6. SAppho (s.d./1998): L'égal des dieux. In: Philippe Brunet, dir. Cent versions d'un poème. Paris: Allia.

\section{RÉFÉRENCES}

Barthes, Roland (1966): Critique et vérité. Tel quel. Paris: Seuil.

Berman, Antoine (1985/1991): La traduction et la lettre ou l'auberge du lointain. Paris: Seuil.

Berman, Antoine, Granel, Gérard, Jaulin, Annick et al. (1985): Les Tours de Babel: essais sur la traduction. Mauzevin: Trans Europ Repress.

Masson, Jean-Yves (2007): Bilan des recherches sur la traduction en littérature comparée. In: Anne Tomiche et Karl Zieger, dir. La Recherche en littérature générale et comparée en France en 2007. Valenciennes: Presses universitaires de Valenciennes.

Nerval, Gérard de (1840/1996): Poèmes d'Outre-Rhin. (Dirigé par Jean-Yves Masson) Paris: Grasset. 